\title{
Doença de Parkinson em idosos: ingestão alimentar e estado nutricional
}

\author{
Parkinson's disease: dietary intake and nutritional status
}

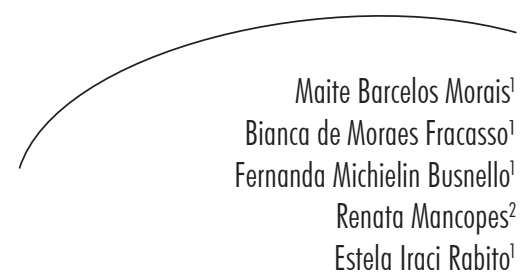

\section{Resumo}

Objetivo: Avaliar o estado nutricional e ingestão alimentar dos idosos portadores da doença de Parkinson. Métodos: Estudo transversal, realizado com pacientes portadores da doença de Parkinson (DP), atendidos em ambulatório. O estado nutricional foi avaliado utilizando a mini-avaliação nutricional (MAN), registro de consumo alimentar estimado de três dias e antropometria. Para avaliação da adequação de ingestão de nutrientes e energia, utilizou-se a ingestão dietética de referência, considerando valores da ingestão adequada, requerimento médio estimado e ingestão dietética recomendada.

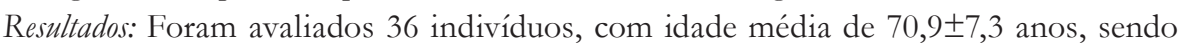
que 55\% dos pacientes apresentaram risco de desnutrição de acordo com a MAN. Todavia, quando classificados pelo índice de massa corporal (IMC), 33\% dos pacientes apresentaram sobrepeso e 39\% não apresentaram perda nos últimos três meses. A ingestão energética foi de 1.632 e $1.840 \mathrm{kcal} / \mathrm{dia}$; proteínas, 71,2 e 61,9g/dia; ferro, 15,8 e $15,6 \mathrm{mg} / \mathrm{dia}$; sódio, 1.798 e $1.843 \mathrm{mg} / \mathrm{dia}$, para homens e mulheres, respectivamente, e ficaram acima das recomendações. Verificou-se ingestão inferior à recomendação para fibras (13,5 e 13,6g/dia), cálcio (694 e 552mg/dia) e potássio (1.637 e $1.476 \mathrm{mg} / \mathrm{dia})$. A ingestão energética apresentou correlação com ferro e proteína $(r=0,581$ e r=0,582) e a proteína apresentou correlação com a ingestão de ferro $(r=0,600)$. Conclusão: Embora a ingestão energética da maioria se apresente adequada, observou-se o desequilíbrio dietético, com consumo insuficiente de fibras, cálcio e potássio. Além disso, metade da população encontrava-se em risco nutricional, segundo a MAN.

\section{Abstract}

Objective: To evaluate the dietary intake of elderly patients with Parkinson's disease and to correlate it with nutritional status. Methods: Cross-sectional study with patients with Parkinson's disease (PD) treated as outpatients. Nutritional status was evaluated by the Mini Nutritional Assessment (MAN) and food intake was assessed by three-day

\footnotetext{
Departamento de Nutrição. Universidade Federal de Ciências da Saúde de Porto Alegre. Porto Alegre, RS, Brasil.

2 Departamento de Fonoaudiologia. Universidade Federal de Santa Maria. Santa Maria, RS, Brasil.

Correspondência / Correspondence

Maite Barcelos Morais

E-mail: maitebarcelos@gmail.com
}

Palavras-chave: Doença de Parkinson. Estado nutricional. Dieta. 
estimated food record. The Dietary Reference Intakes were used to assess the adequacy of nutrients and energy intake, considering adequate intake values, estimated average requirement and recommended dietary allowance. Results: We evaluated 36 subjects with mean age of $70.9 \pm 7.3$ years; $55 \%$ of patients were at risk of malnutrition according to MNA. However, when classified by body mass index (BMI), 33\% of patients were overweight and 39\% had no weight loss in the past three months. Energy intake was 1,632 and 1,840kcal/day; proteins, 71.2 and $61.9 \mathrm{~g} / \mathrm{day}$; iron, 15.8 and $15.6 \mathrm{mg} /$ day; sodium, 1,798 and $1,843 \mathrm{mg} /$ day for men and women, respectively, and were above recommendations. There was less than the recommended intake for fiber (13.5 and 13.6g/day), calcium (694 and 552mg calcium/day), and potassium (1,637 and 1,476mg/ day). Energy intake was correlated with iron and protein $(r=0.581$ and $r=0.582)$ and protein correlated with iron intake $(\mathrm{r}=0.600)$. Conclusion: Although energy intake of the majority was adequate, dietary imbalance was observed, with insufficient intake of fiber, calcium, and potassium. Moreover, half the population was at nutritional risk according to the MNA.
Key words: Parkinson

Disease. Nutritional Status.

Diet.

\section{INTRODUÇÃO}

O envelhecimento da população vem ocorrendo de maneira crescente nos países em desenvolvimento. ${ }^{1}$ As doenças crônicas não transmissíveis, cuja incidência vem aumentando, juntamente com a alteração do perfil demográfico, têm ocupado lugar de destaque no perfil de mortalidade desses países. ${ }^{2}$ A doença de Parkinson (DP) é a segunda doença neurodegenerativa mais comum em idosos, com prevalência estimada de 3,3\% no Brasil, de acordo com o estudo de Barbosa et al.

A doença de Parkinson é uma doença progressiva e degenerativa, em cujo curso a mobilidade do paciente se deteriora, o que pode implicar dificuldades nas atividades de vida diária, tais como fazer compras e cozinhar, o que possivelmente também acarretará mudanças nos hábitos alimentares, ingestão de energia e nutrientes. ${ }^{4-5}$ Poucos estudos têm focado os hábitos alimentares em pacientes com DP. ${ }^{5}$ Além disso, quanto à prevenção da enfermidade aliada a uma alimentação saudável, ainda existem algumas controvérsias, principalmente com relação aos micronutrientes. ${ }^{6}$

Entre as alterações provocadas pela DP, encontra-se a perda de peso como um achado comum. A falta de apetite também está associada a quadros depressivos frequentes, assim como os efeitos colaterais das medicações que também contribuem para a baixa ingestão alimentar, ${ }^{7}$ além das disfunções sensoriais com diferentes intensidades em portadores dessa doença.

Devido à sintomatologia presente, como perda de peso involuntário, dificuldades de mastigação e até mesmo a desnutrição, são necessários cuidados nutricionais específicos, visando a manutenção do peso, ingestão de proteínas adequadas, prevenção do controle de constipação e adaptação do paciente que pode possuir problemas motores. ${ }^{1}$ Além disso, existe grande escassez na literatura de estudos correlacionando a ingestão alimentar de indivíduos com distúrbio do movimento, como a DP. Tendo como base esses fatores, o presente estudo teve como objetivo avaliar o estado nutricional e ingestão alimentar dos idosos portadores da doença de Parkinson.

\section{METODOLOGIA}

Estudo transversal realizado com indivíduos portadores da doença de Parkinson, de ambos os sexos, de 60 a 88 anos de idade, atendidos no ambulatório de Distúrbios do Movimento e Demências do Hospital Santa Clara, do Complexo Hospitalar Santa Casa de Misericórdia de Porto Alegre, RS. 
A amostra adotada neste estudo foi definida por conveniência. Dos 53 pacientes com doença de Parkinson e em uso de L-dopa que foram atendidos no ambulatório, no período de outubro de 2010 a agosto de 2011, foram incluídos 36 pacientes, com 60 anos de idade ou mais. Os critérios de exclusão foram: indivíduos com limitações físicas para realizar as medidas antropométricas, analfabetos sem cuidadores, que pudessem auxiliar na realização do registro de consumo.

A avaliação nutricional foi realizada uma única vez, nos 36 idosos. A primeira parte foi a aplicação da mini-avaliação nutricional (MAN), ${ }^{8}$ que inclui as seguintes medidas antropométricas: peso, ${ }^{9}$ perímetro do braço (PB), ${ }^{10}$ perímetro da panturrilha (PP) ${ }^{10}$ estatura ${ }^{11}$ e cálculo do índice de massa corporal (IMC), classificado segundo Lipschitz. ${ }^{12} \mathrm{O}$ peso foi aferido com balança de plataforma calibrada, da marca Welmy ${ }^{\circledR}$, com peso máximo de $150 \mathrm{~kg}$. A estatura foi estimada através da meia-envergadura, devido a alterações posturais da maioria da população, utilizando-se fita métrica inelástica e inextensível, que também foi utilizada para aferição dos perímetros do braço e da panturrilha.

A segunda parte foi a avaliação de consumo alimentar, realizada por meio do registro de consumo estimado de três dias, ${ }^{13}$ sendo calculados macro e micronutrientes, fibra, cálcio, ferro, sódio, potássio e vitamina $\mathrm{B}_{6}$, bem como o número de refeições realizadas. Os participantes receberam orientações verbais e fotográficas para o preenchimento do registro. Dos 36 pacientes avaliados, apenas 23 apresentaram registro alimentar adequadamente preenchido. Diante disso, a avaliação do consumo e sua correlação com o estado nutricional foram realizadas apenas nesta subamostra.

Os registros foram analisados e as medidas caseiras descritas pelos pacientes, convertidas em gramas e mililitros pelos pesquisadores. Para o cálculo da ingestão, utilizaram-se tabelas de composição alimentar ${ }^{14-15}$ e o software Excel $^{\circledR}$. Para avaliação da adequação de ingestão dos macro e micronutrientes, foram utilizadas as recomendações de ingestão dietética de referência (IDRs) ${ }^{16}$ para idade e sexo, considerando os valores do requerimento médio estimado (RME) para a vitamina $\mathrm{B}_{6}$. A ingestão dietética recomendada (IDR) para os macronutrientes e o ferro, e valores da ingestão adequada (IA) para o cálcio, sódio, fibras e potássio. O requerimento energético foi calculado individualmente pela equação de Harris \& Benedict, com fator atividade 1,3.

As variáveis quantitativas foram descritas por média e desvio-padrão, e as variáveis qualitativas por frequências absolutas e relativas. Para avaliar a associação entre as variáveis qualitativas, os testes qui-quadrado de Pearson foram aplicados. Para comparar médias, o teste $t$ Student foi utilizado. O nível de significância adotado foi de $5 \%$, e o tratamento estatístico dos dados foi realizado por meio do programa SPSS (Statistical Package for the Social Sciences), versão 18.0.

Mediante assinatura do Termo de Consentimento Livre e Esclarecido (TCLE), participantes consentiram em participar da pesquisa, que foi aprovada pelo Comitê de Ética em Pesquisa da Universidade Federal de Ciências da Saúde de Porto Alegre (UFCSPA), projeto ${ }^{\circ}$ 10.681. O estudo não envolveu nenhum risco para os indivíduos e os procedimentos estavam de acordo com as orientações nacionais e internacionais para pesquisas envolvendo seres humanos, estando consoante a Declaração de Helsinki.

\section{RESULTADOS}

Trinta e seis pacientes foram avaliados quanto a antropometria e estado nutricional pela MAN, sendo $58,3 \%$ do sexo masculino. A média de idade da amostra foi de 70,9 $\pm 7,3$ anos.

Os resultados de adequação dos indivíduos em relação às variáveis antropométricas, ingestão hídrica e número de refeições estão descritos na tabela 1. Como esperado, os indivíduos do sexo masculino apresentaram maior peso e estatura, quando comparados com os indivíduos do sexo 
feminino. Com relação ao estado nutricional avaliado pela MAN, 55\% dos idosos apresentaram risco de desnutrição. Todavia, quando classificados de acordo com o IMC, 33\% da amostra apresentaram sobrepeso, sendo que $55 \%$ das mulheres encontravam-se nesta condição. Com relação à perda de peso, 39\% dos indivíduos não apresentaram alteração nos últimos três meses, e $16,6 \%$ não souberam informar.
Quanto à ingestão hídrica, não foram encontradas diferenças significativas entre os sexos. Foi observado que 16,6\% dos indivíduos consumiam menos de três copos de líquidos/ dia, enquanto que $41,7 \%$ consumiam mais de quatro copos de líquidos/dia. Quando avaliado o número de refeições diárias, 36,1\% dos indivíduos realizavam cinco refeições/dia, sendo que $77,7 \%$ das mulheres realizavam cinco ou mais refeições diárias quando comparadas a $64,3 \%$ dos homens.

Tabela 1 - Perfil antropométrico, estado nutricional, ingestão hídrica e $\mathrm{n}^{\circ}$ refeições/dia de portadores da doença de Parkinson, segundo sexo. Porto Alegre-RS, 2011.

\begin{tabular}{lcccc}
\hline \multicolumn{1}{c}{ Variáveis } & $\begin{array}{c}\text { Total } \\
\mathrm{n}=36\end{array}$ & $\begin{array}{c}\text { Homens } \\
\mathrm{n}=21\end{array}$ & $\begin{array}{c}\text { Mulheres } \\
\mathrm{n}=15\end{array}$ & $\mathrm{p}$ \\
\hline Peso $(\mathrm{kg})^{*}$ & $66,9 \pm 11,2$ & $69,7 \pm 10,3$ & $63,2 \pm 11,6$ & 0,086 \\
Estatura $(\mathrm{m})^{*}$ & $1,63 \pm 0,08$ & $1,66 \pm 0,06$ & $1,57 \pm 0,06$ & $<0,001$ \\
$\mathrm{CP}(\mathrm{cm})^{*}$ & $35,3 \pm 5,9$ & $34,5 \pm 7,0$ & $36,4 \pm 4,2$ & 0,340 \\
$\mathrm{CB}(\mathrm{cm})^{*}$ & $29,5 \pm 3,4$ & $29,4 \pm 3,0$ & $29,6 \pm 4,0$ & 0,888 \\
$\mathrm{IMC}\left(\mathrm{kg} / \mathrm{m}^{2}\right)^{* *}$ & $24,4 \pm 4,3$ & $25,08 \pm 3,8$ & $25,7 \pm 4,9$ & 0,669 \\
Estado nutricional (MAN)** & & & & \\
Risco de desnutrição & $20(55,5)$ & $11(30,6)$ & $9(25,0)$ & \\
Desnutrição & $2(5,5)$ & $2(5,5)$ & $0(0)$ & \\
Sem risco de desnutrição & $14(38,8)$ & $8(22,2)$ & $6(16,7)$ & 0,92 \\
$\mathrm{~N}^{\text {o }}$ refeições realizadas/dia* & $4,8 \pm 0,7^{\text {a }}$ & $4,6 \pm 0,5$ & $5,1 \pm 0,8$ & \\
\hline
\end{tabular}

* descrita por média \pm desvio padrão; ** descrita por $n^{\circ}$ absoluto e $\%{ }^{a}{ }^{a} n=23$.

A avaliação do consumo alimentar por meio do registro de consumo está descrita na tabela 2. Apenas 23 registros foram avaliados, pois os demais apresentaram problemas quanto ao preenchimento, estavam ilegíveis ou não foram devolvidos aos pesquisadores.

As quantidades de carboidratos, proteínas e lipídios ingeridos pela amostra apresentavam-se acima dos valores médios recomendados para sexo e idade, ${ }^{16}$ bem como ferro e sódio.Com relação ao consumo de fibras, ambos foram inferiores ao recomendado para a faixa etária, ${ }^{16}$ porém sem diferença significativa entre os sexos. A ingestão de cálcio, potássio e vitamina $\mathrm{B}_{6}$ também foi inferior às recomendações. As mulheres apresentaram ingestão inferior aos homens quanto ao cálcio, potássio e vitamina $\mathrm{B}_{6}$. 
Tabela 2 - Ingestão alimentar média de macro e micronutrientes dos pacientes com doença de Parkinson. Porto Alegre-RS, 2011.

\begin{tabular}{lcc|cc}
\hline & \multicolumn{2}{c}{ Homens } & \multicolumn{2}{c}{ Mulheres } \\
& \multicolumn{1}{c}{$\mathrm{n}=09$} \\
& $\begin{array}{c}\text { Recomendação } \\
\text { Média } \pm \mathrm{DP}\end{array}$ & $\begin{array}{c}\text { Ingerido } \\
\text { Média } \pm \mathrm{DP}\end{array}$ & $\begin{array}{c}\text { Recomendação } \\
\text { Média } \pm \text { DP }\end{array}$ & $\begin{array}{c}\text { Ingerido } \\
\text { Média } \pm \mathrm{DP}\end{array}$ \\
\hline Energia (kcal/dia) & $1780 \pm 592$ & $1632 \pm 554$ & $1711 \pm 270$ & $1840 \pm 336$ \\
Carboidratos (g/dia) & $130,0^{\mathrm{b}}$ & $222,3 \pm 89,4$ & $130,0^{\mathrm{b}}$ & $264,3 \pm 91,1$ \\
Proteínas (g/dia) & $56,0^{\mathrm{b}}$ & $71,2 \pm 15,2$ & $46,0^{\mathrm{b}}$ & $61,9 \pm 11,2$ \\
Lipídios (g/dia)* & 39,5 & $58,9 \pm 19,4$ & 38,0 & $53,2 \pm 9,1$ \\
Fibras (g/dia) & $30,0-38,0^{\mathrm{c}}$ & $13,5 \pm 4,7$ & $21,0-25,0^{\mathrm{c}}$ & $13,6 \pm 6,0$ \\
Cálcio (mg/dia) & $1000-1200^{\mathrm{c}}$ & $694 \pm 287$ & $1000-1200^{\mathrm{c}}$ & $552 \pm 323$ \\
Ferro (mg/dia) & $6,0^{\mathrm{b}}$ & $15,8 \pm 10,1$ & $5,0-8,1^{\mathrm{b}}$ & $15,6 \pm 9,1$ \\
Sódio (mg/dia) & $1200-1500^{\mathrm{c}}$ & $1798 \pm 676$ & $1200-1500^{\mathrm{c}}$ & $1843 \pm 640$ \\
Potássio (mg/dia) & $4700^{\mathrm{c}}$ & $1637 \pm 449$ & $4700^{\mathrm{c}}$ & $1476 \pm 803$ \\
Vitamina B $(\mathrm{mg} /$ dia) & $1,1-1,4^{\mathrm{a}}$ & $1,2 \pm 0,4^{*}$ & $1,1-1,3^{\mathrm{a}}$ & $1,0 \pm 0,5^{*}$ \\
\hline
\end{tabular}

** gramas referentes a 20 a 35\% do VET; ${ }^{\text {a }}=$ EAR para o micronutriente para idade e sexo

${ }^{\mathrm{b}}=$ RDA para o micronutriente para idade e sexo; ${ }^{\mathrm{c}}=$ AI para o micronutriente para idade e sexo.

Quandoavaliados os resultados de correlações de Pearson entre a ingestão de energia, proteína, ferro, cálcio e os dados antropométricos (tabela 3), pode-se observar que existiam evidências de correlação moderadamente positiva entre o consumo energético: ferro e proteína; e entre o consumo proteico e ferro. Não obstante, para as outras variáveis analisadas foram encontradas correlações fracas.

Tabela 3 - Correlação (r) entre a ingestão de energia, proteína, cálcio e ferro e antropometria dos pacientes com doença de Parkinson. Porto Alegre-RS, 2011.

\begin{tabular}{lccccccc}
\hline \multicolumn{1}{r}{$\mathrm{r}$} & Peso & IMC & CB & CP & Ferro & Cálcio & Proteína \\
\hline Energia & 0,341 & 0,254 & 0,053 & 0,140 & 0,581 & 0,410 & 0,582 \\
Proteína & 0,048 & 0,253 & 0,074 & 0,399 & 0,600 & 0,463 & - \\
Cálcio & 0,083 & 0,278 & 0,175 & 0,035 & 0,408 & - & - \\
Ferro & 0,245 & 0,307 & 0,227 & 0,271 & - & - & - \\
\hline
\end{tabular}

$\mathrm{r}=$ correlações de Pearson. 


\section{DISCUSSÃO}

Neste estudo, aproximadamente 55,5\% dos indivíduos apresentavam risco de desnutrição, resultados superiores aos encontrados por Barrichella et al. ${ }^{17} \mathrm{em}$ estudo que encontrou $23 \%$ dos pacientes com DP inicial em risco de desnutrição, quando avaliados pela MAN. A proporção de pacientes com DP em risco de desnutrição aumentou em 11,4\% após investigação longitudinal de três anos. Corroborando os resultados encontrados para prevalência de desnutrição, Wang et al. ${ }^{18}$ e Beyer et al. ${ }^{19}$ também encontraram baixo número de indivíduos desnutridos em seus estudos.

A baixa prevalência de desnutrição pode ser atribuída a vários fatores, incluindo diferenças de raça, idade, tempo de doença e contexto social. No entanto, o fato de a população estudada ter apresentado alta prevalência de risco de desnutrição indica que a DP pode contribuir para o declínio do estado nutricional. No entanto, quando classificado o estado nutricional pelo IMC neste estudo, $81,2 \%$ da amostra foram considerados eutróficos ou sobrepeso. Assim como no estudo de Lorefält et al., ${ }^{20}$ que encontraram valores de IMC de idosos com DP semelhantes aos valores de IMC de idosos saudáveis.

Tendo em vista a transição epidemiológica e o aumento da prevalência do sobrepeso e obesidade na população brasileira, a avaliação isolada de medidas antropométricas e IMC pode disfarçar a presença de risco nutricional. Em contrapartida, essas medidas de fácil obtenção são recomendadas para o monitoramento do estado nutricional.

Assim como neste estudo, as medidas de circunferência do braço e da panturrilha de idosos com DP não se diferenciam das encontradas em idosos saudáveis. ${ }^{21} \mathrm{~A}$ redução de medidas antropométricas resulta, geralmente, de longo prazo de desnutrição, sendo essas medidas pouco sensíveis para detecção precoce de má nutrição. ${ }^{22}$ Diante disso, quando avaliada a associação entre medidas antropométricas e o consumo energético proteico, as mesmas não apresentaram associação, provavelmente por esta população ainda estar em risco nutricional sem manifestações na reserva corporal. $O$ acompanhamento desses pacientes se faz necessário para evitar que mudanças na ingestão alimentar ao longo da evolução da doença possa contribuir para a instalação da desnutrição.

Quando comparada às medidas antropométricas, a MAN é uma ferramenta precisa e sensível de avaliação, devido a sua capacidade abrangente de estimar perda de peso e capacidade funcional, além de incluir medidas antropométricas. Apesar da eficiência da MAN em estimar o estado nutricional de pacientes idosos sob várias condições, ${ }^{23}$ poucos estudos têm avaliado o estado nutricional de pacientes com DP por meio dessa ferramenta, provavelmente por apresentar a limitação de requerer resposta a questões que necessitam de memória, podendo esta estar afetada nessa população.

De acordo com estudo realizado por Beyer et al., ${ }^{19}$ pacientes com doença de Parkinson apresentavam quatro vezes mais propensão a relatar perda de peso quando comparados a indivíduos sadios. Esta mudança pode estar relacionada com alterações metabólicas, sensoriais e aumento do gasto energético, ${ }^{20,24}$ assim como neste estudo, no qual 44\% dos pacientes relataram ter perdido peso nos últimos três meses.

A perda de peso também pode estar relacionada com a ingestão inadequada. Todavia, os valores de ingestão encontrados na amostra, assim como em outros estudos, ${ }^{20,24,25}$ apresentaram adequação na ingestão energética e de macronutrientes nesses indivíduos. Além disso, Lorefalt et al., ${ }^{20} \mathrm{em}$ estudo realizado na Suécia com 26 idosos, demonstraram que os pacientes que apresentavam perda de peso tinham um consumo de energia maior, quando comparados aos pacientes sem perda de peso. O número de refeições realizadas pelos idosos com DP também se assemelhou ao encontrado no estudo sueco (4,9 refeições/dia) e foi superior ao encontrado por Barichella et al. ${ }^{26}$ em estudo realizado em Gana, no qual os portadores de DP faziam em média 2,7 refeições/dia. Não obstante, estes resultados para ingestão de macronutrientes 
são similares aos divulgados pela Pesquisa de Orçamentos Familiares (POF) ${ }^{27}$ 2008/2009, tanto para a população brasileira, quanto para a população da Região Sul.

Neste estudo, os pacientes apresentaram baixa ingestão de fibras, quando comparadas às recomendações ${ }^{16}$ ao consumo da população brasileira, e da Região Sul do país, ${ }^{27}$ e também inferiores aos encontrados por Ådén et $\mathrm{al}^{21} \mathrm{e}$ Barrichella et al. ${ }^{26} \mathrm{~A}$ baixa ingestão de fibras pode ser fator de risco para constipação, que nesta doença pode chegar a 80\% dos pacientes. ${ }^{28,29}$ A constipação intestinal é motivo de grande desconforto entre os parkinsonianos e tem se encontrado relação entre presença e grau de constipação com a duração e gravidade da doença. ${ }^{1}$ Associada a esses fatores, também está a baixa ingestão hídrica apresentada por esses indivíduos. Gage et al. ${ }^{28}$ observaram que $61,2 \%$ dos indivíduos com DP apresentavam uma ingestão hídrica de cinco a oito copos de líquidos por dia, quando comparados ao encontrado nesta amostra.

O consumo de ferro mostrou-se maior, quando comparado ao consumo nacional e da Região Sul, ${ }^{27}$ e esteve correlacionado ao consumo proteico e energético dos pacientes portadores de Parkinson. O ferro pode aumentar a produção de radicais livres, resultando em estresse oxidativo, com degeneração dos neurônios dopaminérgicos na doença de Parkinson. ${ }^{30}$ Além disso, em estudo realizado por Powers et al., ${ }^{6}$ constatou-se que existe forte associação entre o ferro ingerido na dieta e a doença de Parkinson.

Corroborando os resultados apresentados por Lorefält et al., ${ }^{20}$ bem como os divulgados pela $\mathrm{POF}^{27}$ o consumo de cálcio foi abaixo da recomendação de ingestão adequada (IA) para o micronutriente. ${ }^{16} \mathrm{O}$ cálcio é um mineral envolvido em muitos processos metabólicos e encontrado principalmente no leite e seus derivados. Em estudo realizado em Honolulu com 7.504 homens japoneses com idade entre 45 e 68 anos, durante 30 anos, buscou-se a relação entre a ingestão de leite e produtos lácteos com o risco de desenvolvimento da doença de Parkinson. O resultado demonstrou que a incidência da doença de Parkinson aumentava nos homens que consumiam leite e produtos lácteos, em comparação com os que não consumiam. Tal associação, no entanto, ainda não está bem definida; os autores acreditam que o leite e seus derivados, ou os contaminantes presentes no leite, como substâncias utilizadas na fabricação de pesticidas, podem alterar a absorção de componentes neuroprotetores com funções antioxidantes, induzindo o surgimento da doença de Parkinson nesses indivíduos. ${ }^{31}$

A deficiência de vitamina $\mathrm{B}_{6}$ pode contribuir para o aumento da hiper-homocisteínemia. Lewerin et al. ${ }^{32}$ concluíram que a prevalência de hiper-homocisteinemia encontrouse inversamente correlacionada com $\mathrm{O}$ desenvolvimento motor e a performance cognitiva na doença de Parkinson. Além disso, estudo recente ${ }^{33}$ tem discutido o papel da neurotoxicidade da homocisteína sobre o tecido nervoso na doença de Parkinson. Comparando os valores de recomendação da ingestão diária de $\mathrm{B}_{6}$, observa-se adequação da ingestão desta vitamina, mas quando comparada à ingestão da população brasileira $^{27}$ e à ingestão de idosos suecos com $\mathrm{DP}^{21}{ }^{21}$ a ingestão de $\mathrm{B}_{6}$ desta amostra foi menor.

Assim como no presente estudo, Tomazoni et al. ${ }^{34}$ identificaram baixo consumo de potássio em idosos, relacionando esse resultado ao baixo consumo de alimentos integrais, frutas e vegetais relatados por meio de um questionário de frequência alimentar, diferentemente de Ådén et al. $^{21}$ Aliado ao baixo consumo de potássio, encontrou-se elevado consumo de sódio, assim como no estudo de Ådén et al. ${ }^{21}$ e como observado por Sarno et al. ${ }^{35}$ em estudo com a população brasileira, no qual idosos excederam em quase duas vezes o limite recomendado.

Como limitação do presente estudo, cabe afirmar que os valores de ingestão encontrados, no entanto, podem ser subestimados ou superestimados, uma vez que o registro pode alterar o consumo alimentar e o relato das medidas caseiras pode ter sido inadequado, bem como a estimativa energética por meio de equações pode ter subestimado o gasto energético dos portadores de DP. 


\section{CONCLUSÕES}

Embora o consumo energético-proteico apresente-se adequado quando comparado às necessidades estimadas, observou-se a ingestão insuficiente de fibras, cálcio, ferro e potássio, e excessiva de sódio. Associada a este perfil dietético, a maioria dos idosos apresentou risco nutricional, mas os dados antropométricos ainda se assemelham aos de idosos saudáveis.

\section{REFERÊNCIAS}

1. Farhud CC, Marucci MFN. Avaliação dietética de indivíduos com doença de Parkinson. Gerontology 2004;12(1-2):16-21.

2. Cervato AM, Derntl AM, Latorre MRDO, Marucci MFN. Educação nutricional para adultos e idosos: uma experiência positiva em Universidade Aberta da Terceira Idade. Rev Nutr 2005;18(1):41-52.

3. Barbosa MT, Caramelli P, Maia DP, Cunningham MC, Guerra HL, Lima-Costa MF, et al. Parkinsonism and Parkinson's disease in the elderly: a communitybased survey in Brazil (The Bambui study). Mov Disord 2006;21(6):800-8.

4. Wylie C, Copeman J, Kirk SFL. Health and social factors affecting the food choice and nutritional intake of elderly people with restricted mobility. J Hum Nutr Diet 1999;12:375-80.

5. Andersson I, Sidenvall B. Case studies of food shopping, cooking and eating habits in older women with Parkinson's disease. J Adv Nurs 2001;35(1):69-78.

6. Powers KM, Smith-Weller T, Franklin GM, Longstreth WT Jr, Swanson PD, Checkoway H. Parkinson's disease risks associated with dietary iron, manganese, and other nutrient intakes. Neurology 2003;60(11):1761-6.

7. D’Oliveira FA, Frank AA, Soares EA. A influência dos minerais na doença de Parkinson. Nutrire Rev Soc Bras Aliment Nutr 2007;32(1):77-88.

8. Guigoz Y. The Mini Nutricional Assessment (MNA) review of the literature -- What does it tell us? J Nutr Health Aging 2006;10(6):466-85.

9. Organización Mundial de la Salud. El estado físico: uso e interpretación de la antropometria. Informe de un Comité de Expertos de la OMS. Serie de informes técnicos 854. Genebra: WHO; 1995.
Os resultados apresentados neste estudo, portanto, precisam ser interpretados com cautela. É necessário realizar estudos com maior número de pacientes com doença de Parkinson e comparar os resultados com os do grupo controle de indivíduos não portadores da doença. Além disso, o acompanhamento desta população durante a evolução da doença poderá contribuir para o entendimento do processo de desnutrição que está associado à doença.

10. Frisancho AR. Anthropometric standards: an interactive nutritional reference of body size and body composition for children and adults. 4th ed. Ann Arbor, Michigan: University of Michigan Press; 2011.

11. Mitchell CO, Lipschitz DA. Arm length measurement as an alternative to height in nutritional assessment of the elderly. JPEN J Parenter Enteral Nutr 1982;6(3):226-9

12. Lipschitz DA. Screening for nutritional status in the ederly. Prim Care 1994;21(1):55-67.

13. Fisberg RM; Slater B; Machioni DML, Martini LM. Inquéritos alimentares: métodos e bases científicas. 2. ed. Barueri: Manole; 2005

14. Philippi ST. Tabela de Composição de Alimentos: Suporte para Decisão Nutricional. 2. ed. São Paulo: Coronário; 2002.

15. Pinheiro ABV, Lacerda EMA, Benzecry EH, Gomes MCS, Costa VM. Tabela para Avaliação de Consumo Alimentar em Medidas Caseiras. 5. ed. Rio de Janeiro: Atheneu; 2000.

16. Institute of Medicine; Food and Nutrition Board. Dri Dietary reference intakes: applications in dietary assessment. Washington: National Academy Press; 2001.

17. Barichella M, Villa MC, Massarotto A, Cordara SE, Marczewska A, Vairo A, et al. Mini Nutritional Assessment in patients with Parkinson's disease: correlation between worsening of the malnutrition and increasing number of disease-years. Nutr Neurosci 2008;11(3):128-34.

18. Wang G, Wan Y, Cheng Q, Xiao Q, Wang Y, Zhang $\mathrm{J}$, et al. Malnutrition and associated factors in Chinese patients with Parkinson's disease: results from a pilot investigation. Parkinsonism Relat Disord 2010;16(2):119-23. 
19. Beyer PL, Palarino MY, Michalek D, Busenbark K, Koller WC. Weight change and body composition in patients with Parkinson's disease. J Am Diet Assoc 1995;95(9):979-83.

20. Lorefält B, Ganowiak W, Wissing U, Granérus AK, Unosson M. Food habits and intake of nutrients in elderly patients with Parkinson's disease. Gerontology 2006;52(3):160-8.

21. Ådén E, Carlsson M, Poortvliet E, Stenlund H, Linder J, Edström M, et al. Dietary intake and olfactory function in patients with newly diagnosed Parkinson's disease: a case-control study. Nutr Neurosci 2011;14(1):25-31.

22. Christensson L, Unosson M, Ek AC. Evaluation of nutritional assessment techniques in elderly people newly admitted to municipal care. Eur J Clin Nutr 2002;56(9):810-8.

23. Guigoz Y, Lauque S, Vellas BJ. Identifying the ederly at risk for malnutrition. The Mini Nutritional Assessment. Clin Geriatr Med 2002;18(4):737-57.

24. Quagliato LB, Viana MA, Quagliato EMAB, Simis S. Alterações do olfato na doença de Parkinson. Arq Neuro-psiquiatr 2007,65(3a):647-52.

25. Marczewska A, De Notaris R, Sieri S, Barichella M, Fusconi E, Pezzoli G. Protein intake in Parkinsonian patients using the EPIC food frequency questionnaire. Mov Disord 2006;21(8):1229-31.

26. Barichella M, Akpalu A, Cham M, Privitera G, Cassani E, Cereda E, et al. Nutritional status and dietary habits in Parkinson's disease patients in Ghana. Nutrition 2013;29(2):470-3.

27. Instituto Brasileiro de Geografia e Estatística. Pesquisa de Orçamentos Familiares 2008-2009: análise do consumo alimentar pessoal do Brasil. Rio de Janeiro: IBGE; 2011.

28. Gage H, Kaye J, Kimber A, Storey L, Egan M, Qiao Y, et al. Correlates of constipation in people with Parkinson's. Parkinsonism Relat Disord 2011;17(2):106-11.

29. Pfeiffer RF. Gastrointestinal dysfunction in Parkinson's disease. Lancet Neurol 2003;2(2):107-16.

30. Kaur D, Andersen J. Does cellular iron dysregulation play a causative role in Parkinson's disease? Ageing Res Rev 2004;3(3):327-43.

31. Park M, Ross GW, Petrovitch H, White LR, Masaki $\mathrm{KH}$, Nelson JS, et al. Consumption of milk and calcium in midlife and the future risk of Parkinson disease. Neurology 2005;64(6):1047-51.

32. Lewerin C, Matousek M, Steen G, Johansson B, Steen B, Nilsson-Ehle H. Significant correlations of plasma homocysteine and serum methylmalonic acid with movement and cognitive performance in elderly subjects but no improvement from short-term vitamin therapy: a placebo-controlled randomized study. Am J Clin Nutr 2005;81(5):1155-62.

33. Bottiglieri T, Diaz-Arrastia R. Hyperhomocysteinemia and cognitive function: more than just a casual link? Am J Clin Nutr 2005;82(3):493-4.

34. Tomazoni T, Siviero J. Consumo de potássio de idosos hipertensos participantes do Programa Hiperdia do município de Caxias do Sul, RS. Rev Bras Hipertens 2009;16(4):246-50.

35. Sarno F, Claro RM, Levy RB, Bandoni DH, Ferreira SRG, Monteiro CA. Estimativa de consumo de sódio pela população brasileira, 2002-2003. Rev Saúde Pública 2009;43(2):219-25. 